Case Report

\title{
A Case Report of Recurrent Takotsubo Cardiomyopathy in a Patient during Myasthenia Crisis
}

\author{
Anusha Battineni, ${ }^{1}$ Naresh Mullaguri, ${ }^{1}$ Shail Thanki, ${ }^{1}$ \\ Anand Chockalingam, ${ }^{2}$ and Raghav Govindarajan ${ }^{1}$ \\ ${ }^{1}$ Department of Neurology, University of Missouri, 1 Hospital Drive, Columbia, MO 65212, USA \\ ${ }^{2}$ Department of Cardiology, University of Missouri, 1 Hospital Drive, Columbia, MO 65212, USA \\ Correspondence should be addressed to Raghav Govindarajan; govindarajanr@health.missouri.edu
}

Received 9 July 2017; Revised 8 September 2017; Accepted 27 September 2017; Published 19 October 2017

Academic Editor: Michael J. Cawley

Copyright (C) 2017 Anusha Battineni et al. This is an open access article distributed under the Creative Commons Attribution License, which permits unrestricted use, distribution, and reproduction in any medium, provided the original work is properly cited.

\begin{abstract}
Introduction. Patients with myasthenia crisis can develop Takotsubo stress cardiomyopathy (SC) due to emotional or physical stress and high level of circulating catecholamines. We report a patient who developed recurrent Takotsubo cardiomyopathy during myasthenia crisis. Coexisting autoimmune disorders known to precipitate stress cardiomyopathy like Grave's disease need to be evaluated. Case Report. A 69-year-old female with seropositive myasthenia gravis (MG), Grave's disease, and coronary artery disease on monthly infusion of intravenous immunoglobulin (IVIG), prednisone, pyridostigmine, and methimazole presented with shortness of breath and chest pain. Electrocardiogram (ECG) showed ST elevation in anterolateral leads with troponemia. Coronary angiogram was unremarkable for occlusive coronary disease with left ventriculogram showing reduced wall motion with apical and mid left ventricle (LV) hypokinesis suggestive of Takotsubo stress cardiomyopathy. Her symptoms were attributed to MG crisis. Her symptoms, ECG, and echocardiographic findings resolved after five cycles of plasma exchange (PLEX). She had another similar episode one year later during myasthenia crisis with subsequent resolution in 10 days after PLEX. Conclusion. Takotsubo cardiomyopathy can be one of the manifestations of myasthenia crisis with or without coexisting Grave's disease. These patients might benefit from meticulous fluid status and cardiac monitoring while administering rescue treatments like IVIG and PLEX.
\end{abstract}

\section{Introduction}

Myasthenia gravis (MG) is a progressive autoimmune neuromuscular junction disorder that affects skeletal muscle leading to considerable morbidity and rarely mortality. Myasthenia crisis is defined as acute worsening of weakness in bulbar and respiratory muscles requiring mechanical ventilation [1]. The primary mode of treatment is immunomodulation with high-dose oral steroids, intravenous immunoglobulin (IVIG), and plasma exchange (PLEX) to clear the acetylcholine receptor autoimmune antibodies, which are responsible for neuromuscular junction dysfunction [1]. Myasthenia patients may have other coexisting autoimmune diseases like thyroiditis and polymyositis which can impact their disease course and treatment [2]. Myasthenia crisis patients may have high levels of circulating catecholamines due to physical and emotional stress, which can cause stress-induced cardiomyopathy [3-6]. There have been several case reports of patients developing Takotsubo stress cardiomyopathy (SC) with left ventricular (LV) dysfunction during myasthenia crisis but its recurrence is rare [7]. We are reporting a patient with seropositive MG and Grave's disease who developed different variants of SC one year apart during subsequent myasthenia crisis posing a significant challenge to administering immunomodulatory treatments.

\section{Case Report}

A 69-year-old Caucasian female with seropositive myasthenia gravis on monthly maintenance intravenous immunoglobulin ( $1 \mathrm{gram} / \mathrm{kg}$ body weight) every 4 weeks and prednisone $50 \mathrm{mg}$ PO daily and pyridostigmine $60 \mathrm{mg}$ three times a day was transferred to the university hospital cardiology service with acute onset chest pain, palpitations, nausea, 
TABLE 1: Cardiac abnormalities and thyroid function during two episodes of myasthenia gravis crisis.

\begin{tabular}{lcccc}
\hline MG crisis & TSH mcunit/ml & Peak troponin, ng/ml & ECG changes & Echo findings \\
\hline Episode 1 & 7.6 & 0.16 & ST elevation V1-V6, Q waves V1-V3 & $\begin{array}{c}\text { Mid ventricular variant Takotsubo stress } \\
\text { cardiomyopathy with reduced ejection } \\
\text { fraction (EF) of 25\% }\end{array}$ \\
\hline Episode 2 & 0.569 & 0.32 & Deep, symmetric T inversions V1-V6 & $\begin{array}{c}\text { Mid and apical akinesia, EF 30\% consistent } \\
\text { with classical apical ballooning type of } \\
\text { Takotsubo stress cardiomyopathy }\end{array}$ \\
\hline
\end{tabular}

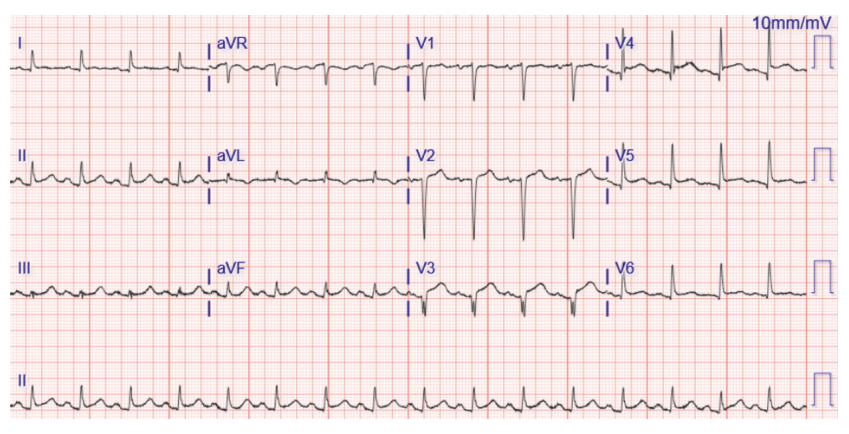

FIGURE 1: ECG during first episode of Takotsubo stress cardiomyopathy with ST elevation V1-V6, Q waves in V1-V3.

and profuse sweating on waking up in the morning at a long-term acute care (LTAC) facility. The symptoms did not relieve with multiple doses of sublingual nitrate. Her medical history is significant for hypertension, coronary artery disease status after myocardial infarction one year earlier with stent placement in left anterior descending artery (LAD), chronic obstructive pulmonary disease with a recent exacerbation with respiratory failure requiring tracheostomy, Grave's ophthalmopathy on oral methimazole $10 \mathrm{mg}$ daily, and 2 packs per day smoking history. She also complained of worsening double vision, slurred speech with nasal quality, difficulty with swallowing her own saliva, and generalized weakness. Her physical examination revealed severe ophthalmoparesis in horizontal and vertical directions, worsening bilateral proptosis, facial diplegia with drooling, and nasal intonation. Wheezing was noted in the upper lobes as well as crepitations in bilateral basal regions. Cardiovascular examination revealed S3 gallop, no raised jugular venous pressure, and regular pulses. Neurological exam was consistent with generalized weakness in proximal and distal muscles with easy fatigability and absent deep tendon reflexes. Her initial ECG showed ST elevations in the anteroseptal leads (V1-V4) (Figure 1) and troponin elevation concerning for ST elevation myocardial infarction (STEMI) and she had emergent cardiac catheterization. No new coronary lesions were detected and prior stent was patent. Left ventriculogram suggested severe LV dysfunction and transthoracic echocardiogram (TTE) confirmed mid ventricular variant Takotsubo stress cardiomyopathy with reduced ejection fraction (EF) of $25 \%$ (Figure 2 and Table 1). Her TSH was elevated to 7.6 from $2.3 \mathrm{micro}$ unit $/ \mathrm{ml}$ with elevated anti-thyroglobulin and antithyroid peroxidase (TPO) autoantibodies suggesting worsening of Grave's disease for which endocrinology recommended continuing high-dose oral steroids (Table 1). Neurology team was consulted for myasthenia crisis management, and they recommended PLEX and opined that the crisis was probably secondary to Grave's disease and methimazole that was started about 3 weeks earlier. She was managed conservatively with resolution of all her symptoms, ECG findings, and troponemia with 5 cycles of PLEX and was discharged back to the LTAC. The repeat TTE 4 weeks from the day of admission showed complete resolution of left ventricular dysfunction. She was continued on oral prednisone $50 \mathrm{mg}$ daily and monthly IVIG (1 gram/kg body weight). One year later, she had a similar episode of ophthalmoparesis, generalized weakness with acute chest pain, troponemia with deep $\mathrm{T}$ wave inversions in V1-V6 leads on ECG (Figure 3) with TTE showing mid and apical akinesia, and EF 30\% consistent with classical apical ballooning type of Takotsubo stress cardiomyopathy (Table 1). Her Grave's disease was well controlled with normal TPO autoantibodies and TSH (Table 1). We diagnosed her with recurrent Takotsubo stress-induced cardiomyopathy secondary to myasthenia crisis and treated her with 5 cycles of PLEX with complete resolution of the ST elevations (Figure 4) and troponemia and she was discharged to rehabilitation facility. Repeat TTE 3 weeks later showed complete normalization of LV function and wall motion (EF $60 \%)$.

\section{Discussion}

Myasthenia gravis is an autoimmune disorder of the neuromuscular junction predominantly in the skeletal muscles. Patients with this disease develop myasthenia crisis due to various reasons like infection, medications, and coexisting autoimmune diseases [1]. This condition and its management add significant amount of physical and psychological stress predisposing patients towards Takotsubo or stress cardiomyopathy [2-7]. Stress cardiomyopathy is a well-known complication associated with neurointensive care patients with subarachnoid hemorrhage, stroke, traumatic brain injury, and status epilepticus [8]. Rarely, certain neuromuscular junction disorders like myasthenia crisis also precipitate SC probably secondary to increased levels of circulating catecholamines [3-6, 9]. The commonest form is apical ballooning syndrome characterized by apical and mid wall akinesis and hypercontractile basal left ventricular segments [10]. Mid and basal LV segments or focal variants are also increasingly recognized now [10]. Coexistence of myasthenia gravis and Grave's disease in patients is well known and sometimes difficult to manage as treatment of one disease can worsen the 


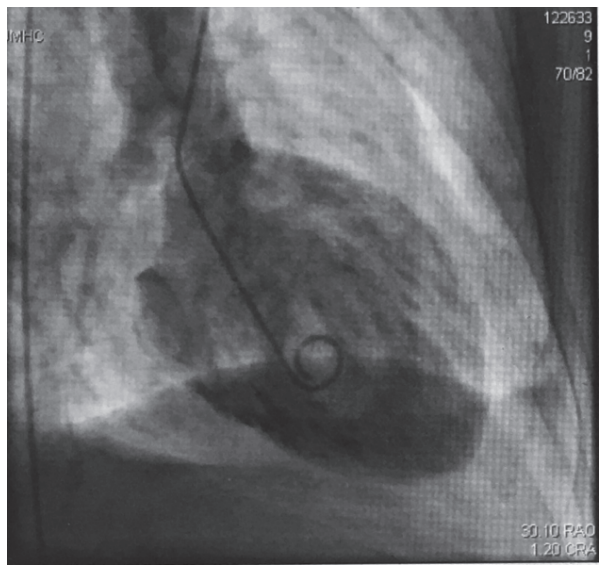

(a)

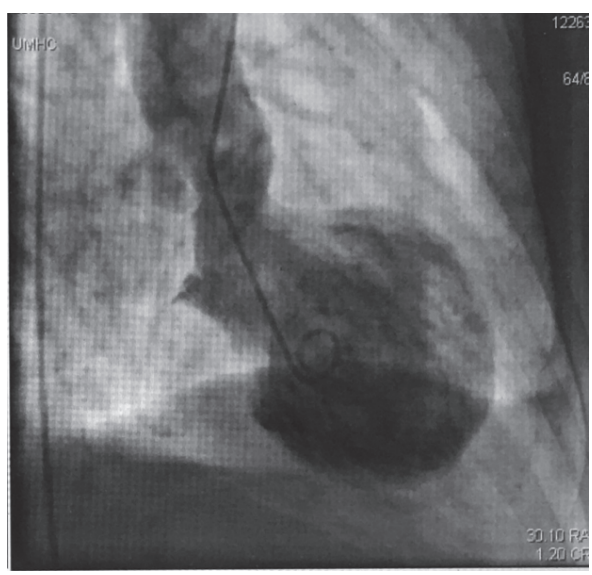

(b)

FiguRE 2: Left ventriculogram in RAO projection in diastole (a) demonstrates normal cavity contour and in systole (b) preserved basal and apical contractility with akinesia of the mid ventricle consistent with Takotsubo stress cardiomyopathy.

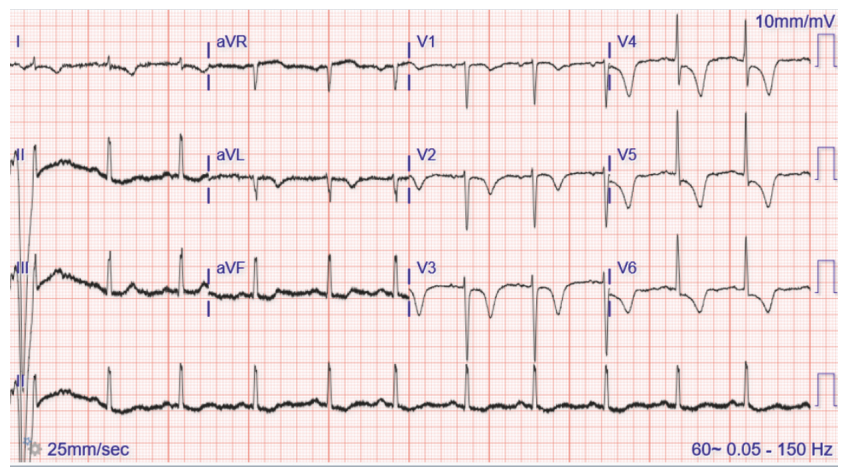

FIGURE 3: ECG during second SC episode with deep symmetrical T inversions in leads V1-V6.

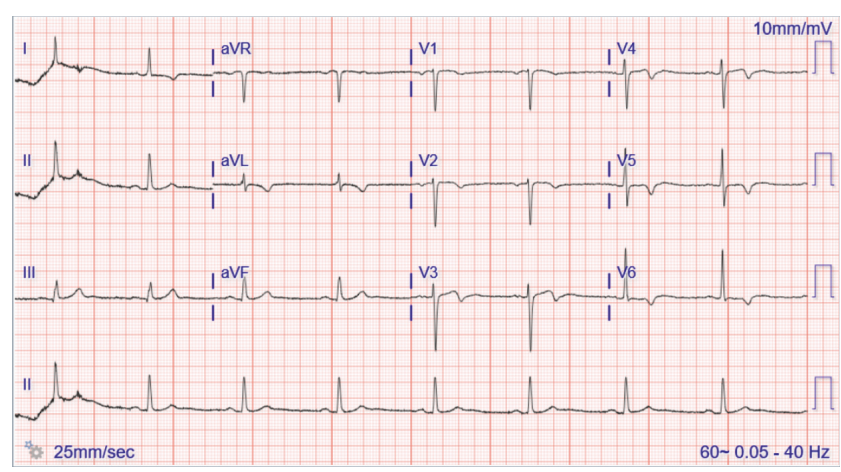

FIGURE 4: ECG 3 days later showing nonspecific ST-T wave changes only.

other like in our patient $[2,11-17]$. Three weeks prior to her first episode of SC, she had worsening of her Grave's disease as manifested by proptosis and rising levels of TPO autoantibodies for which methimazole (10 mg daily) was started. Methimazole can unmask an underlying neuromuscular junction disorder in patient with Grave's disease or trigger myasthenia crisis in patients with coexisting myasthenia gravis [18]. Both Grave's disease and myasthenia crisis were independently known to cause Takotsubo cardiomyopathy $[19,20]$. In our patient, the combination of hyperthyroidism and methimazole might have triggered myasthenia crisis and subsequently SC. Over a dozen case reports describe Takotsubo cardiomyopathy complicating hyperthyroidism [19]. Coexisting coronary artery disease could cause chronic LV dysfunction with remodeling/thinning or new wall motion changes with ischemia. These factors add to the complexity, but SC can be diagnosed confidently in many instances based on carefully evaluating and recognizing SC-induced characteristic wall motion abnormalities $[8,10]$. LV dysfunction improved after both diseases were managed appropriately but required prolonged hospitalization. She was continued on maintenance monthly IVIG infusions $(1 \mathrm{gram} / \mathrm{kg}$ body weight) and oral prednisone (50 mg daily) and, one year later, she developed subsequent crisis and recurrence of SC. We did not find any significant trigger for her crisis after an extensive evaluation and her symptoms resolved completely with PLEX in 10 days. We think her subsequent episode of SC resolved quickly compared to the first episode as her Grave's disease was well controlled. We propose that MG patients in crisis should be screened for other autoimmune disorders like Grave's disease, which can affect neuromuscular junction and cardiac muscle. Neuromuscular specialists and intensivists managing myasthenia crisis patients should be aware of SC. If patient's cardiac symptoms like chest pain, troponemia, or ischemic ECG change during crisis, early recognition of SC and coronary artery disease can improve prognosis [20]. IVIG is contraindicated in patients with low ejection fraction as it may cause fluid overload and precipitate congestive heart failure [21]. In case reports by Gautier et al. and Anand et al., IVIG has been even shown to trigger Takotsubo cardiomyopathy $[22,23]$. Cardiac complications are also known with PLEX in patients with MG with or without Takotsubo [24]. Thus, careful monitoring of fluid status and cardiac function with either IVIG or PLEX is recommended. This is especially so in older patients and in those with severe 
myasthenia, as they are particularly susceptible to Takotsubo cardiomyopathy and the risk of associated congestive heart failure, prolonged hospitalization, and its associated complications with either rescue treatment [20].

\section{Conclusion}

MG patients may have other autoimmune disorders like Grave's disease, which can worsen neuromuscular junction dysfunction and precipitate crisis. Patients who develop Takotsubo stress cardiomyopathy during myasthenia crisis require early recognition of characteristic wall motion patterns and screening for other causes like hyperthyroidism to avoid prolonged hospitalization with increased morbidity and mortality. Meticulous cardiac and fluid monitoring especially in older patients and with severe myasthenia might be beneficial in these patients as they might be at increased risk for developing recurrent stress cardiomyopathy.

\section{Abbreviations}

MG: Myasthenia gravis

ECG: Electrocardiogram

TTE: Transthoracic echocardiogram

IVIG: Intravenous immunoglobulin

PLEX: Plasma exchange

LV: Left ventricle

TPO: Thyroid peroxidase.

\section{Consent}

Written informed consent was obtained from the patient for publication of this case report and any accompanying images.

\section{Disclosure}

An approval from the ethics committee is not applicable to publish this case report. Data sharing is not applicable to this case report as no data sets were generated or analyzed during the current study. Anusha Battineni, Naresh Mullaguri, and Shail Thanki are co-first authors.

\section{Conflicts of Interest}

Anusha Battineni (M.B.B.S.), Naresh Mullaguri (M.D.), Shail Thanki (M.D.), Anand Chockalingam (M.D.), and Raghav Govindarajan (M.D.) do not report any conflicts of interest.

\section{Authors' Contributions}

Anusha Battineni (M.B.B.S.), Naresh Mullaguri (M.D.), Shail Thanki (M.D.), Anand Chockalingam (M.D.), and Raghav Govindarajan (M.D.) contributed to case report design, data collection, and manuscript writing. All authors read and approved the final manuscript. The authors contributed equally to writing this case report and formatting the images.

\section{References}

[1] L. C. Wendell and J. M. Levine, "Myasthenic crisis," The Neurohospitalist, vol. 1, no. 1, pp. 16-22, 2011.

[2] T. Kobayashi, H. Asakawa, Y. Komoike, Y. Nakano, Y. Tamaki, and M. Monden, "A patient with graves' disease, myasthenia gravis, and polymyositis," Thyroid, vol. 7, no. 4, pp. 631-632, 1997.

[3] V. Bansal, M. M. Kansal, and J. Rowin, "Broken heart syndrome in myasthenia gravis," Muscle \& Nerve, vol. 44, no. 6, pp. 990993, 2011.

[4] S. R. Beydoun, J. Wang, R. L. Levine, and A. Farvid, "Emotional stress as a trigger of myasthenic crisis and concomitant takotsubo cardiomyopathy: a case report," Journal of Medical Case Reports, article 393, 2010.

[5] S. Bijulal, S. Harikrishnan, N. Namboodiri, V. K. Ajitkumar, D. Gupta, and P. S. Mathuranath, "Tako-tsubo cardiomyopathy in a patient with myasthenia gravis crisis: a rare clinical association," BMJ Case Reports, 2009.

[6] J. Finsterer and C. Stöllberger, "Stress from myasthenic crisis triggers Takotsubo (broken heart) syndrome," International Journal of Cardiology, vol. 203, pp. 616-617, 2016.

[7] C. P. Wong and P. L. Chia, "Recurrent takotsubo cardiomyopathy precipitated by myasthenic crisis," International Journal of Cardiology, vol. 155, no. 1, pp. el1-e12, 2012.

[8] A. Chockalingam, "Stress cardiomyopathy of the critically ill: Spectrum of secondary, global, probable and subclinical forms," Indian Heart Journal, 2017.

[9] I. B. Harries, H. Levoir, C. Bucciarelli-Ducci, and S. Ramcharitar, "Takotsubo cardiomyopathy in myasthaenia gravis crisis confirmed by cardiac MRI," BMJ Case Reports, 2015.

[10] A. Chockalingam, G.-Y. Xie, and K. C. Dellsperger, "Echocardiography in stress cardiomyopathy and acute lvot obstruction," The International Journal of Cardiovascular Imaging, vol. 26, no. 5, pp. 527-535, 2010.

[11] S. Chhabra and B. Pruthvi, "Ocular myasthenia gravis in a setting of thyrotoxicosis," Indian Journal of Endocrinology and Metabolism, vol. 17, no. 2, pp. 341-343, 2013.

[12] D. M. Jacobson, "Acetylcholine receptor antibodies in patients with graves' ophthalmopathy," Journal of Neuro-Ophthalmology, vol. 15, no. 3, pp. 166-170, 1995.

[13] J. Kubiszewska, B. Szyluk, P. Szczudlik et al., "Prevalence and impact of autoimmune thyroid disease on myasthenia gravis course," Brain and Behavior, vol. 6, no. 10, article e00537, 2016.

[14] S. Kumar, "Management of ocular myasthenia gravis coexisting with thyroid ophthalmopathy," Neurol India, vol. 51, no. 2, author reply 284, pp. 283-284, 2003.

[15] K. Lakhal, Y. Blel, M. Fysekidis, K. Mohammedi, and L. Bouadma, "Concurrent Graves disease thyrotoxicosis and myasthenia gravis: The treatment of the former may dangerously reveal the latter," Anaesthesia, vol. 63, no. 8, pp. 876-879, 2008.

[16] D. G. Mappouras, G. Philippou, S. Haralambous et al., "Antibodies to acetylcholinesterase cross-reacting with thyroglobulin in myasthenia gravis and Graves' disease," Clinical \& Experimental Immunology, vol. 100, no. 2, pp. 336-343, 1995.

[17] M. Marinó, R. Ricciardi, A. Pinchera et al., "Mild clinical expression of myasthenia gravis associated with autoimmune thyroid diseases," The Journal of Clinical Endocrinology \& Metabolism, vol. 82, no. 2, pp. 438-443, 1997.

[18] M. Baretić, S. Balić, and G. Gudelj, "Myasthenic crisis as a side effect of methimazole therapy: Case report," Acta clinica Croatica, vol. 49, no. 1, pp. 67-71, 2010. 
[19] A. E. Omar S, H. Mazek, T. Mahmoud, S. Soontrapa, and J. Suarez, "Takotsubo cardiomyopathy associated with hyperthyroidism treated with thyroidectomy," Baylor University Medical Center Proceedings, vol. 28, no. 2, 2015.

[20] P. Shivamurthy and M. W. Parker, "Cardiac manifestations of myasthenia gravis: a systematic review," IJC Metabolic and Endocrine, vol. 5, pp. 3-6, 2014.

[21] A. A. Vo, V. Cam, M. Toyoda et al., "Safety and adverse events profiles of intravenous gammaglobulin products used for immunomodulation: a single-center experience," Clinical journal of the American Society of Nephrology, vol. 1, no. 4, pp. 844852, 2006.

[22] P. Gautier, R. Ravan, M. Najjar et al., "Tako-Tsubo syndrome during normal human immunoglobolin perfusion," Annales de Cardiologie et d'Angéiologie, vol. 60, no. 5, pp. 290-295, 2011.

[23] U. S. Anand, S. Viswanathan, and J. Arulneyam, "Pulmonary edema in myasthenic crisis," Case Reports in Critical Care, vol. 2013, Article ID 863620, 3 pages, 2013.

[24] M. Arai, H. Ukigai, and H. Miyata, "A case of transient left ventricular ballooning ("Takotsubo"- shaped cardiomyopathy) developed during plasmapheresis for treatment of myasthenic crisis," Journal of Clinical Neurology, vol. 44, no. 3, pp. 207-210, 2004. 


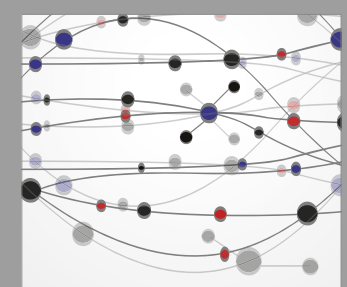

The Scientific World Journal
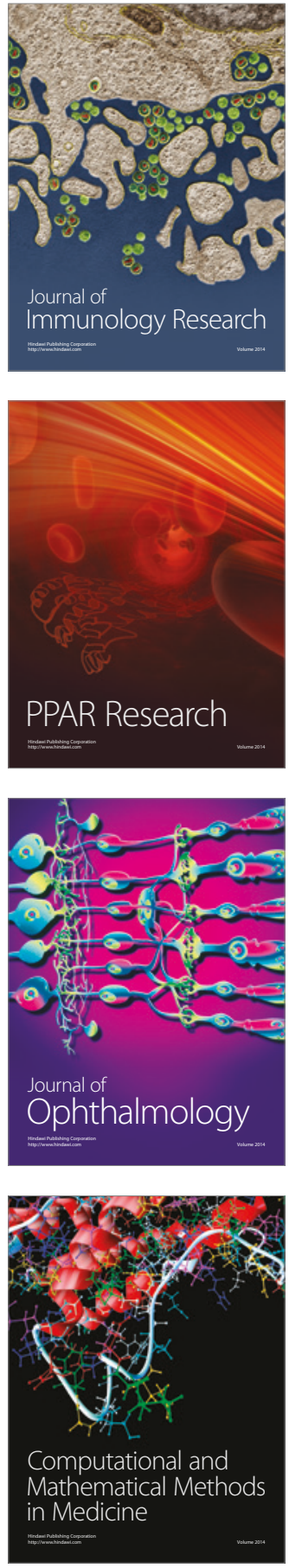

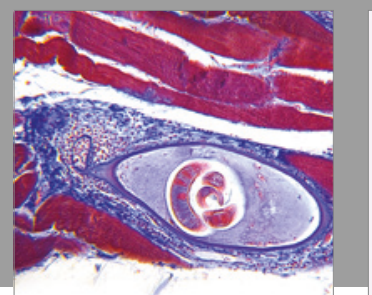

Gastroenterology Research and Practice
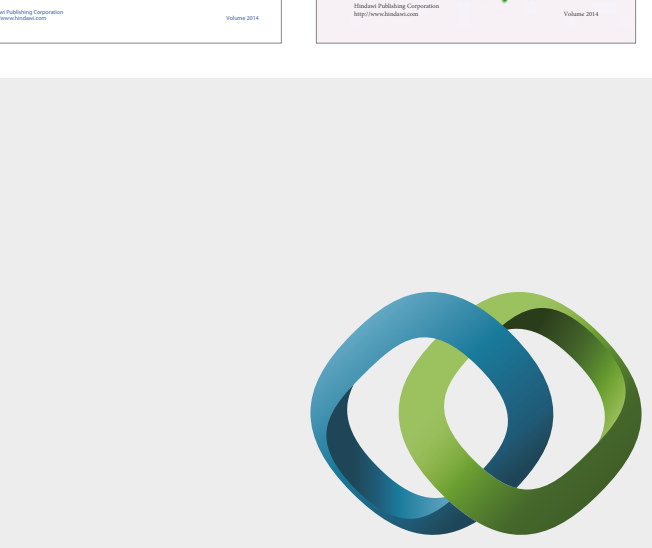

\section{Hindawi}

Submit your manuscripts at

https://www.hindawi.com
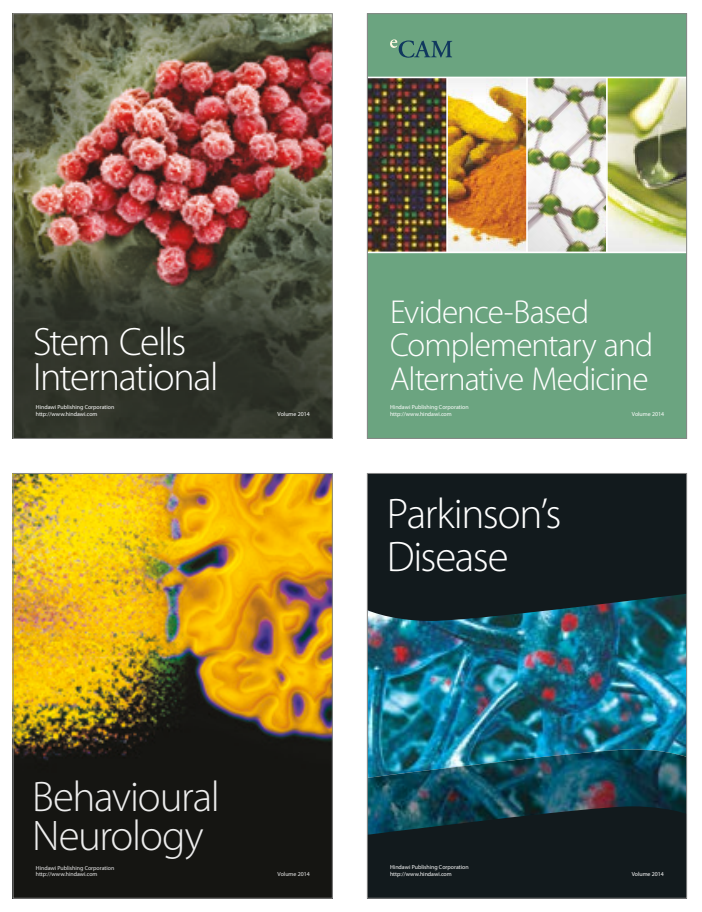
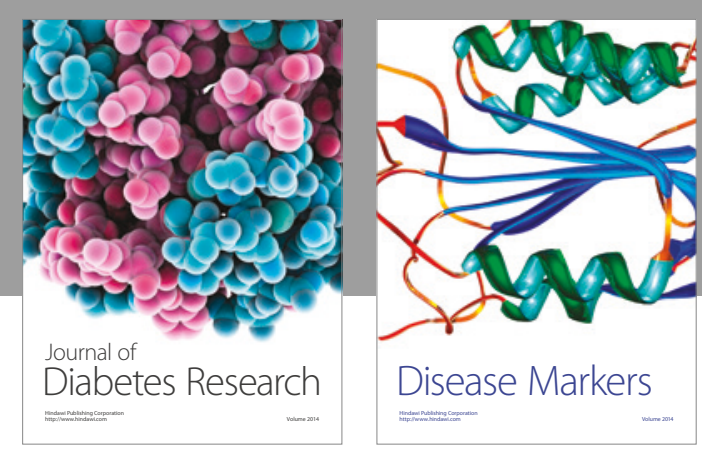

Disease Markers
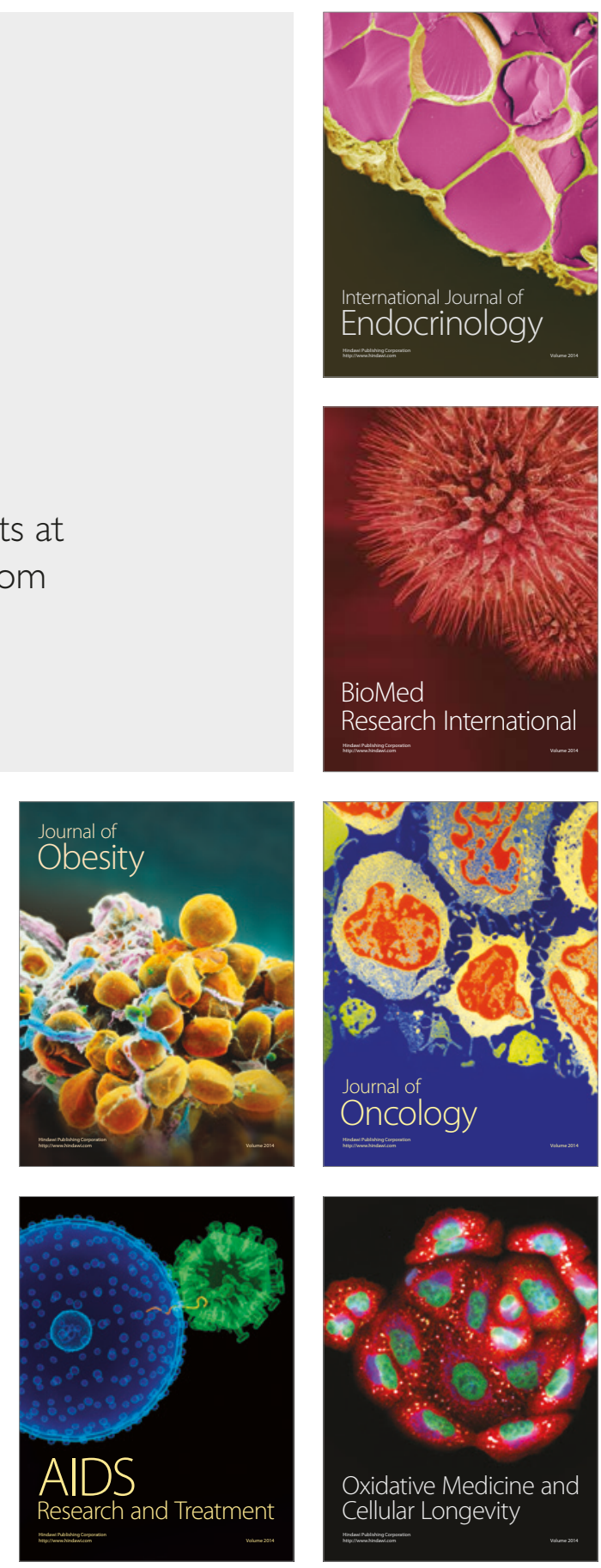